\title{
Mutations in two neuroblastoma rat sarcoma oncogenes are associated with progression of haematologic malignancies in Nigeria
}

\author{
Nneoma Confidence JeanStephanie Anyanwu ${ }^{\mathrm{a}, \mathrm{b}^{*}}$, Ahmed Babangida Suleiman ${ }^{\mathrm{a}}$, Elijah Ekah Ella ${ }^{\mathrm{a}}$, \\ Haruna Makanjuola Kazeem ${ }^{c}$, Maryam Aminu ${ }^{a}$ \\ a Department of Microbiology, Faculty of Life Sciences, Ahmadu Bello University, Zaria, Nigeria \\ ${ }^{b}$ Department of Biological Sciences, Bingham University, P.M.B. 005, Karu, Nigeria \\ 'Department of Veterinary Microbiology, Faculty of Veterinary Medicine and Public health, Ahmadu Bello University, Zaria, Nigeria
}

Received 7th January 2021 / Accepted 4th May 2021

\begin{abstract}
Although mutation in the $R A S$ genes has become important in the evaluation of haematologic malignancies worldwide, developing countries like Nigeria are yet to integrate it as a diagnostic tool and prognostic indicator for improved therapy. This study determined mutations in codons 12 and 13 of NRAS gene in blood donors and haematologic malignant individuals using multiplex (AS-PCR) and Sanger sequencing, thus highlighting the mutations as helpful diagnostic and prognostic tool. AS-PCR was used to selectively amplify mutation-specific synthetic oligonucleotides from the cfDNA of 100 study participants (50 cancer patients and 50 blood donors). Percentage mutation of $31.0 \%$ was seen in NRAS_G12D gene while NRAS_G13C had 17.0\%. Twenty nine $(29.0 \%)$ of the NRAS_G12D mutations were found in haematopoietic malignant patients and $2.0 \%$ were found in blood donors, while $15.0 \%$ of the NRAS_G13C were found in the malignant patients, confirming the occurrence of NRAS gene mutations in haematologic cancers and predominance of the G-A transition. The highest rate of mutation was observed in leukaemia patients, having a significant association with codon $13(\mathrm{p}=0.042)$. Stages 3 and 2 cancers each had the highest mutation rates of NRAS_G12D and NRAS_G13C, revealing possible link between these mutations and susceptibility and progression of haematologic malignancies, which is higher in leukaemia. Further NRAS mutation studies and its role in other cancers are advocated, especially targeted towards ameliorating diagnosis and prognostic therapy. Challenges related to diagnosis and management of haematologic cancer continue to persist in developing countries like Nigeria. Thus, there is a need to go beyond studying the incidence and distribution pattern of these malignancies to capturing immunogenetic parameters of affected individuals.
\end{abstract}

Keywords: cfDNA, cancer, leukaemia, mutation, NRAS_G12D, NRAS_G13C

\section{INTRODUCTION}

Cancer is the leading cause of death globally (WHO, 2019) and constitutes more than 200 distinctively different genetic diseases which include the 3 major groups of haematologic malignancies (multiple myeloma, leukaemia and lymphoma) (Anyanwu et al., 2019). Cancer has emerged as a major public health problem in developing countries. Between now and 2030, about 13 million deaths will be caused by cancer if there is no intervention. About 16 million new cases were diagnosed in 2020 (Anyanwu et al., 2019), $70 \%$ of which would be from developing

\footnotetext{
*Author for correspondence: Nneoma Confidence JeanStephanie Anyanwu, Department of Microbiology, Faculty of Life Sciences, Ahmadu Bello University, Zaria, Nigeria. Postal Code: 810107. Email anyanwunneomaconfidence@gmail.com
} 
countries (WHO, 2019; GBDCC, 2017). Following the gradual enlightenment on this bane of mortality/morbidity in the country, certain number of studies are recently being carried out to determine the epidemiology of the disease in certain regions of Nigeria (Onwuasigwe et al., 2002; Omoti and Halim, 2006; Omoti and Imiere, 2006; Nwannadi et al., 2010; Omoti et al., 2012; Egesie et al., 2018). Lymphoma and leukaemia are the sixth and seventh most common types of cancer in Nigerian population. Immunological risk factors may be involved in a complex interplay of genetic damage in somatic cells caused by chronic antigenic stimulation, cytokine dysregulation and defective mutations resulting in development of these malignancies (Hamid, 2012). Rat sarcoma ( $R A S$ ) proteins are Guanosine triphosphatases (GTPases) that regulate pathways responsible for proliferation and cell survival. Mutations of the $\mathrm{R} A S$ gene lead to the uncontrolled growth factor independent cellular proliferation of hematopoietic progenitors (Liang et al., 2018). The activation of multiple signalling pathways like RAS/RAF/MAPK and the phosphoinositide 3-kinase (PI3K) plays an important role in regulating cell cycle progression, cell growth and proliferation, angiogenesis, migration, cytoskeletal changes, apoptosis, and senescence. Thus, the accumulation of mutations in tumour suppressor genes and participating proto-oncogenes such as NRAS significantly contributes to the progression of haematopoietic cancer (McCubrey et al., 2006).

The burden of cancer is increasing in economically developing countries as a result of population aging and growth as well as, increasingly, an adoption of cancer-associated lifestyle choices including smoking and alcohol intake, physical inactivity, and feeding habit. Despite the increase in the occurrence of bloodrelated oncogenic viruses and haematologic malignancies in the country (Egesie et al., 2017) studies on cancer are still largely limited to prostate, cervical, and breast cancers. Most of these studies focus on the pattern of the diseases, the presentation and the prevalence (Anyanwu et al., 2019), thus, there is lack of immunogenetic information on these diseases. This study is one of the very few to explore NRAS mutations in Nigerians.
Challenges related to diagnosis and management of haematologic cancer continue to persist in developing countries like Nigeria, thus the need to go beyond studying the incidence and distribution pattern of these malignancies to capturing immunogenetic parameters of affected individuals. This need was met by this study through providing important insight on the overall burden of the diseases, which in turn is hoped to inform haematopoietic cancer control strategies and provide a footstool for investigating underlying determinants of these diseases, given that they are important causes of morbidity and mortality in Nigeria. Exploring the immunogenetics of malignant patients in this study exposed a helpful diagnostic tool which is hitherto sparsely used in Nigeria.

\section{MATERIALS AND METHODS}

\section{Study area}

Federal Capital Territory (FCT), Abuja, Nigeria constitutes the study area. Fifty haematologic cancer patients and fifty blood donors, diagnosed in three selected hospitals, were sampled. The selected hospitals include University of Abuja Teaching Hospital, Gwagwalada, National Hospital, Abuja, and Garki Hospital, Abuja, Nigeria.

\section{Study population and design}

A hospital-based cross-sectional study was carried out among patients between the ages of 7-71 years old recruited from the Oncology Unit and the Blood Bank Unit of these hospitals. Random probability sampling technique was employed for blood donors while purposive sampling was used for cancer patients.

\section{Inclusion and exclusion criteria}

Individuals from the target population who gave informed consent and using the services of the selected hospitals at the time of the study were included, while pregnant women, extremely anaemic patients $(<70 \mathrm{~g} / \mathrm{l})$ and any individual who indicated interest after the maximum number of samples were obtained were excluded. 


\section{Sample collection and processing}

Anonymized sociodemographic data, laboratory data, clinical data (where applicable) and $2-3 \mathrm{ml}$ of blood plasma samples were obtained from fifty blood donors and fifty haematopoietic cancer patients from June 2018 to August 2019. Sociodemographic data were obtained from each participant using questionnaires. Sample collection was done with the aid of hospital personnel. Blood plasma were separated into two aliquots of at least $1 \mathrm{ml}$. Healthy blood donors underwent prior HBV, HCV, HIV and VDRL screening. The HMP were those already enrolled in chemotherapy, as well as newly diagnosed ones showing clinical manifestations and confirmed using complete blood count (CBC), bone marrow and/or lymph node aspiration and biopsy, positron emission tomography - computed tomography (PET-CT) scan and magnetic resonance imaging (MRI) by the hospitals. The blood plasma samples were stored immediately at $-70^{\circ} \mathrm{C}$ until further processing at the Department of Microbiology, Faculty of Life Sciences, Ahmadu Bello University, Zaria, Nigeria.

Cell-free DNA was isolated from blood plasma with Plasma/Serum Cell-Free Circulating DNA Purification Mini $\mathrm{Kit}^{\mathrm{TM}}$ (Norgen Biotek Corp, Canada \#55100), following manufacturer's protocol. NRAS mutation was determined via Allele-Specific (AS) PCR technique and Sanger Sequencing technique.

\section{RAS gene amplification and AS-PCR for NRAS}

A PCR volume of $20 \mu \mathrm{l}$ containing the extracted DNA was amplified with the primers and conditions previously described (Anyanwu et al., 2019). The AS-PCR amplifications of the NRAS gene were performed in a volume of $20 \mu \mathrm{l}$ for ASP $12(\mathrm{G}>\mathrm{A})$ and CYS $(\mathrm{G}>\mathrm{T}) \mathrm{NR} A S$ mutations, respectively, using the modified version of the technique by (Vasioukhin et al., 1996) and the primers and conditions previously described by (Anyanwu et al., 2019). The details of the target mutations are shown in the Table 1 below:

\section{Sanger sequencing}

The amplified NRAS PCR products were sent to Inqaba Biotec, South Africa, for sequencing. Sanger sequencing technique was used to further detect single nucleotide variants (SNVs) using the PCR amplification products, after purification. The products were purified using NucleoFast 96 PCR Clean-up Kit (Macherey-Nagel) according to the manufacturer's instructions. Seven $\mu \mathrm{L}$ of the purified products were then used for the sequencing reaction using the BigDye Terminator v1.1 Cycle Sequencing Kit (Applied Biosystems Inc., Foster City, California, USA). Sequencing reaction products were purified before electrophoresis using the Montage SEQ96 Sequencing Reaction Kit (Millipore Corporation). Sequencing analysis was performed on the Applied Biosystems Genetic Analyzer (DNA Sequencer, the ABI Model 3500XL).

The results obtained from the Sanger sequencing platform were then analysed for heterogeneous mutations (SNVs) using the Mutation Surveyor ${ }^{\mathrm{TM}}$ and Unipro UGENE ${ }^{\mathrm{TM}}$ Bioinformatics software version 5.1.2 and 33.0, respectively. For Mutation Surveyor ${ }^{\mathrm{TM}}$, the electropherograms were tested against the NRAS reference sequence (NCBI, NM_002524.5) which served as the wild type. For UGENETM, the chromatograms were analysed with the reference gene from the National Center for Biotechnology Information (NCBI) BLAST database. Further confirmation was done by converting the synthetic chromatogram files (.scf) to FASTA sequences using ClustalX ${ }^{\mathrm{TM}}$ software version 2.1 (University College, Dublin) and subsequently blasting the converted FASTA directly on the NCBI database to observe mismatches.

\section{Statistical analysis}

Mutation analysis was evaluated with SPSS version 20 (IBM SPSS Incorporated, Chicago, IL, USA). Baseline data were presented using descriptive statistics. Chi-Square or Fisher's exact test, where adequate, were used to analyse the relationship between the mutations and different groups/clinic-demographic factors. Dichotomous variables between each factor was compared using Chi-Square. Results were considered statistically significant at $95 \%$ confidence interval $(P<0.05)$. 
Table 1. Background information on target mutations.

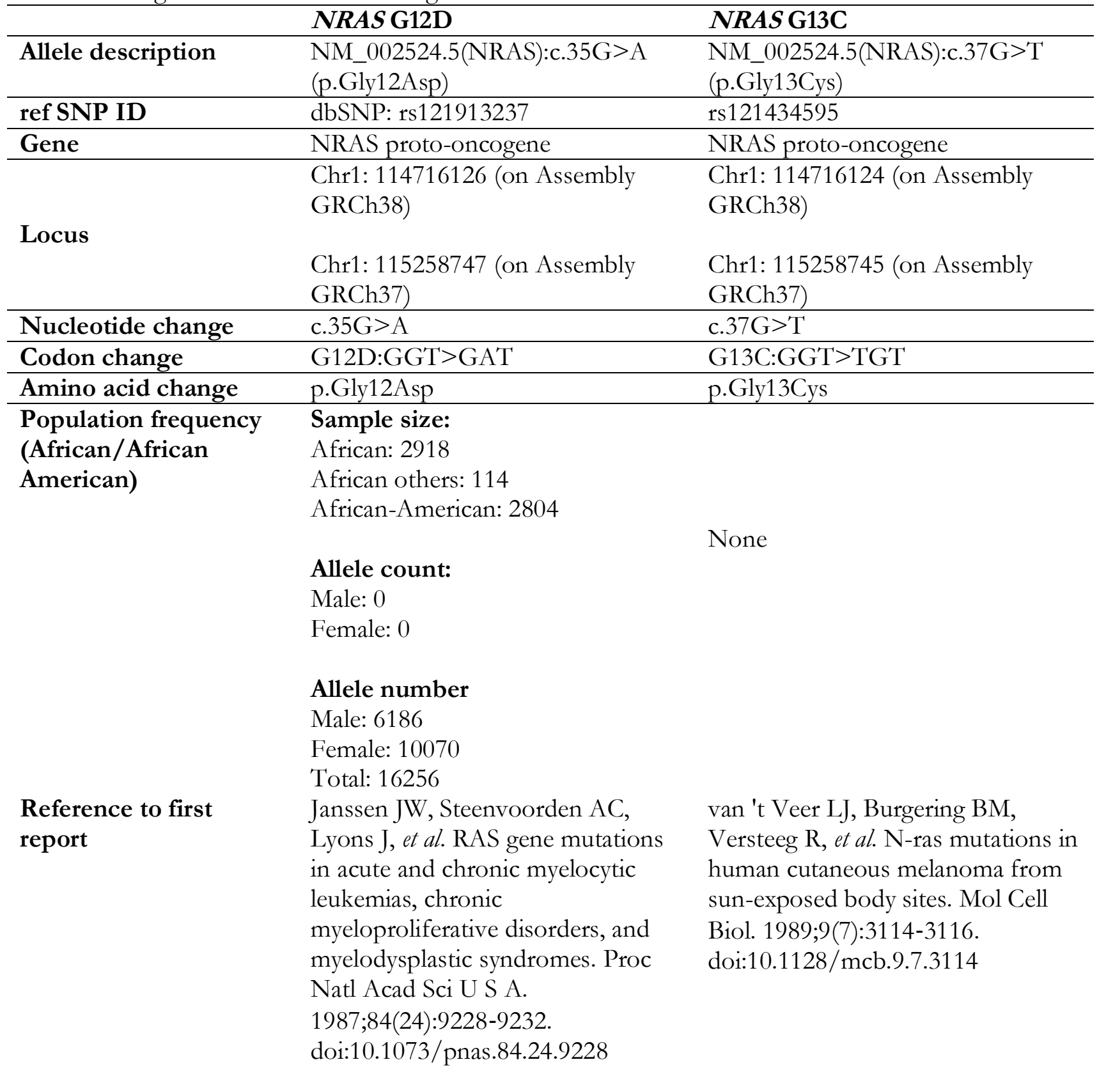

\section{RESULTS}

The total number of samples examined for NRAS mutation was 100 , comprising of 50 cancer patients and 50 healthy blood donors. Out of these, $27(27.0 \%)$ male and $23(23.0 \%)$ female cancer patients were examined while $41(41.0 \%)$ male and $9(9.0 \%)$ female blood donors were included in the mutation analysis. The study population was aged 7-71 years. The mean age
$( \pm \mathrm{SD})$ was $30.70( \pm 12.572)$. The proportion of HMP according to histological subtypes was 16.0\% (MM), 14.0\% (ALL, CML, NHL), 10.0\% (AML, CLL, HL), $4.0 \%$ (TCL) and $2.0 \%$ (CLL/SLL, CMML, MPN, PCM), respectively. The gender distribution of these haematological malignancies among the cancer patients is displayed in Figure 1. While the highest rate of cancer was seen among male patients with ALL and $\mathrm{MM}$, the highest rate of cancer among female patients was seen in those with CML and NHL. 
No cases of CLL/SLL, CMML, PCM and TCL were seen among female patients, while no cases of MPN was observed among male patients.

Out of the 100 samples analysed, NRAS mutations were found in $48(48.0 \%)$, out of which $4(4.0 \%)$ were blood donors. The overall mutations for NRAS_G12D and NRAS_G13C were $31(31.0 \%)$ and $17(17.0 \%)$ each, while $52.0 \%$ of the study participants had no mutation (Table 2). Out of the 31 mutations on codon 12, a total of $29(29.0 \%)$ cell-free DNA were from cancer patients while $2(2.0 \%)$ were blood donor samples. For the NRAS_G13C codon, 15 $(15.0 \%)$ patients and $2(2.0 \%)$ blood donors had the mutation, respectively. No mutation was found in 6 haematologic malignant patients (Table 2).

The relationship between the two NRAS mutations and risk factors, demographic and clinical features among the malignant patients is presented in Table 3. No NRAS_G13C mutations were observed among patients below 20 years of age, but the highest mutation rate was seen in participants below 20 years for NRAS_G12D. However, the highest rate of NRAS_G13C was seen in patients within the age groups 21-30 and 41-50, respectively. There was no association between the age of the study participants and the NRAS gene mutation (ASP: $\mathrm{p}=0.226$; CYS: $\mathrm{p}=0.158)$. Out of the 27 male patients, $16(32.0 \%)$ had point mutation on codon 12 while $7(14.0 \%)$ had point mutation on codon 13. For the female patients, transition was observed in $13(26.0 \%)$ patients on codon 12 while transversion on codon 13 was observed in 8 (16.0) patients. Although there was no association between mutation and gender (ASP: $p=0.845$; CYS: $\mathrm{p}=0.496$ ), point mutation was observed to be higher among males (Table 3 ). The highest rate of mutation in both codons was observed among leukaemia patients. There was significant association between NRAS_G13C mutation and malignancy type $(p=0.042)$, but there was no significant relationship between the type of malignancy and NRAS_G12D mutation. There was also no significant association between both mutations and the stages of cancer (ASP: $\mathrm{p}=$ 0.369; CYS: $p=0.284$ ), although the highest rates were seen in stages 3 and 2 for NRAS_G12D and NRAS_G13C each. Stage 2 cancer had the highest frequency of $24.0 \%$ (24), while stage 4 cancer had the lowest frequency $(2,2.0 \%)$. Stages 1 and 3 had $4.0 \%$ and $20.0 \%$, respectively. Five $(10.0 \%)$ of the cancer patients were HIV positive (Table 3).

The relationship between NRAS mutations and all the risk factors analysed among the malignant patients were statistically insignificant (Table 3). Twenty four percent $(24.0 \%)$ of the patients who had NRAS_G12D had a history of blood donation/reception, $12.0 \%$ had a history of substance abuse, $14.0 \%$ have had STDs in the past, and $18.0 \%$ have a history of cancer in their family and previous radiotherapy/X-ray. For NRAS_G13C, $16.0 \%$ of the patients had a history of blood donation/reception, $8.0 \%$ had a history of substance abuse, $12.0 \%$ have had STDs in the past, and $10.0 \%$ have a history of cancer in their family and previous radiotherapy/X-ray.

The relationship between NRAS gene mutations, and demographic and risk factors among the blood donors is shown on Table 4. The rate of mutation with respect to all the analysed factors was $\leq 4.0 \%$. There was no association between NRAS mutation and the analysed factors.

Heterogeneous mutations were observed for NRAS_G12D (c.35 GGT>GAT) via Sanger sequencing. However, the NRAS_G13C (c.37 $\mathrm{G}>\mathrm{T}$ ) mutations which were observed through AS-PCR were not detected by sequencing. A variation in the nucleotide " $\mathrm{A}$ " at position 35, from the " $G$ " present in the reference gene denotes NRAS G12D (Figures 2 and 3).

Table 2. Distribution of NRAS_G12D and NRAS_G13C mutations in codons 12 and 13 among study participants $(\mathrm{N}=100)$.

\begin{tabular}{cccc}
\hline \multirow{2}{*}{ Mutation Status } & \multicolumn{3}{c}{ Frequency (\%) } \\
\cline { 2 - 4 } & Malignant Patients & Blood Donors & Total \\
\hline NRASS_G12D & $29(29.0)$ & $2(2.0)$ & $31(31.0)$ \\
NRASS_G13C & $15(15.0)$ & $2(2.0)$ & $17(17.0)$ \\
No mutation & $6(6.0)$ & $46(46.0)$ & $52(52.0)$ \\
Total & $50(50.0)$ & $50(50.0)$ & $100(100.0)$ \\
\hline
\end{tabular}




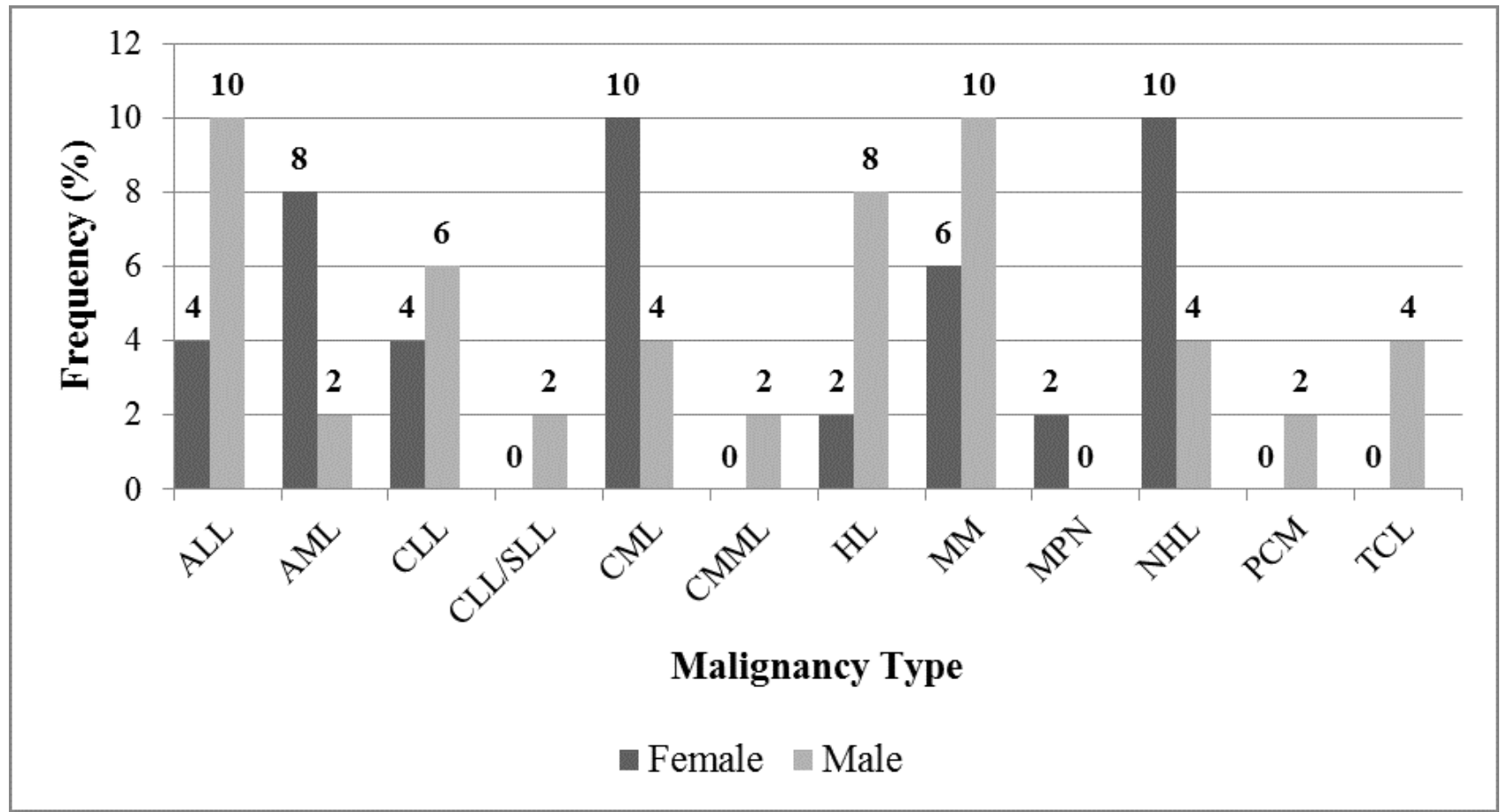

Figure 1. Distribution of haematologic cancer among malignant individuals. Figure shows occurrence of 12 cancer subtypes among malignant patients. Fisher's Exact test value $=14.674, \mathrm{p}=0.128$. Key: $\mathrm{MM}=$ multiple myeloma; CLL/SLL = Chronic lymphocytic lymphoma/small lymphocytic lymphoma; ALL = acute lymphocytic leukaemia, $\mathrm{AML}=$ acute myelogenic leukaemia; $\mathrm{CLL}=$ Chronic lymphocytic leukaemia; $\mathrm{CML}=$ chronic myelogenic leukaemia; $\mathrm{TCL}=\mathrm{T}$-cell lymphoma; $\mathrm{PCM}=\mathrm{Plasma}$ cell myeloma; $\mathrm{MPN}=$ myeloproliferative neoplasm; $\mathrm{CMML}=$ chronic myelomonocytic leukaemia; NHL = non-Hodgkin lymphoma; HL = Hodgkin lymphoma.
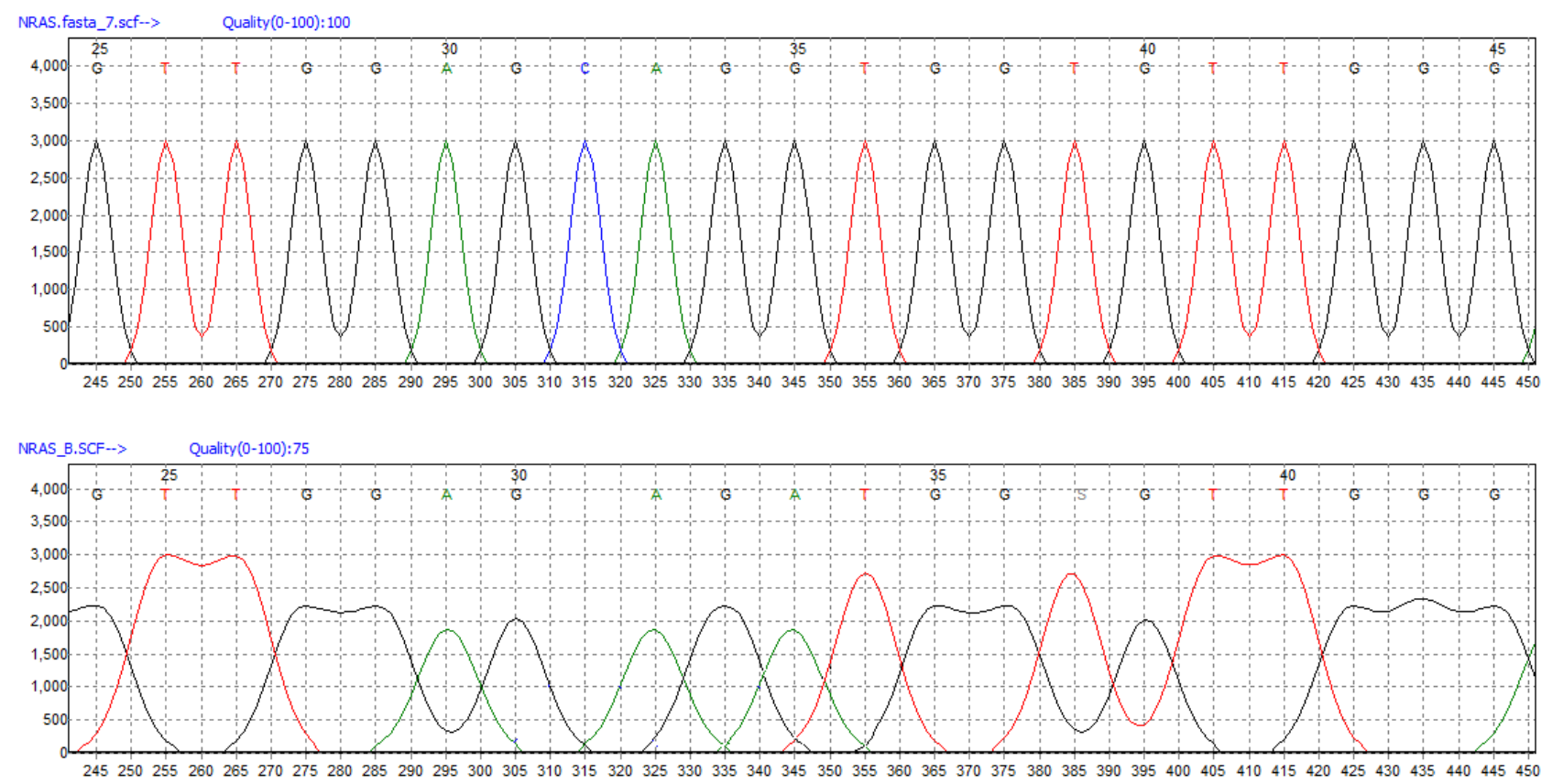

Figure 2. Sequence Chromatogram showing NRAS_G12D Mutation at Position 35 (c.35G>A). Figure shows electropherogram from Mutation Surveyor ${ }^{\mathrm{TM}}$ software with NRAS_G12D mutation at Position 35 of codon 12 in the NRAS gene. 


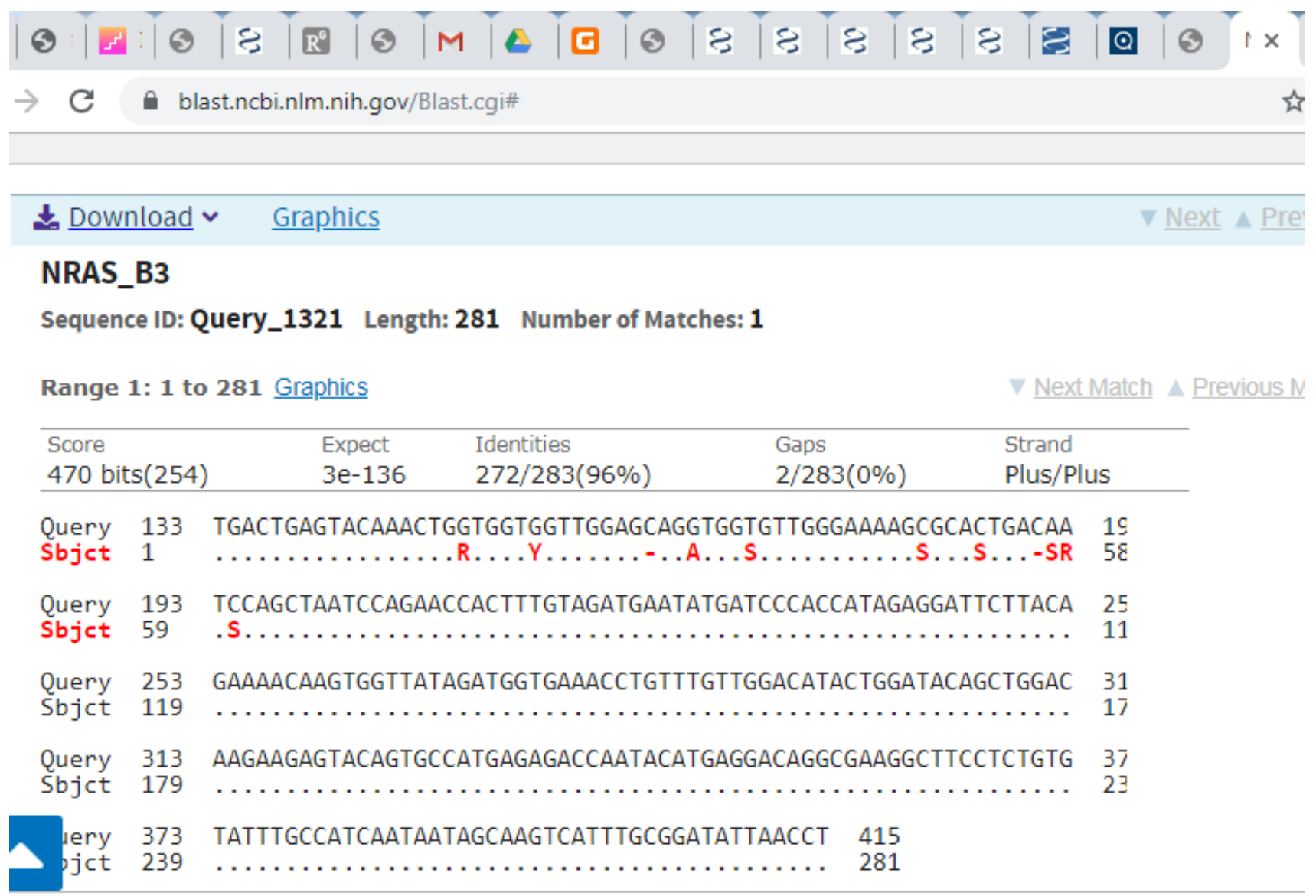

Figure 3. NCBI Blast Result Showing Target Mutation (c.35G>A) alongside other mutations. Figure shows Blast result of NRAS_G12D mutation against the NRAS reference sequence from NCBI database (NCBI, NM_002524.5).

\section{DISCUSSION}

Haematologic malignancies are responsible for significant morbidity and mortality of affected individuals, with a higher rate in developing countries. The EGFR signalling plays a key role in the development and progression of cancer as it triggers downstream signalling cascades (Anyanwu et al., 2019), including the RAS-RAFMAPK pathway, to stimulate cell proliferation, differentiation, survival and invasion (Anyanwu et al., 2019). Detection of mutation in haematologic malignant patients is therefore of great importance in Nigerian setting to better understand the molecular biology of the diseases, and for better risk assessment of individual patients, as well as for better prognostic therapy. NRAS mutation rates vary widely in hematopoietic cancers, including leukaemia (CML, CMML, Juvenile
Myelomonocytic Myeloid Leukaemia (JMML)), lymphoma and multiple myeloma (plasma cell myeloma). However, G12D (i.e. NRAS_G12D) mutations generally prevail over those of G13C (i.e. NRAS_G13C) (Fernández-Medarde and Santos, 2011).

Overall, multiple myeloma had the preponderant frequency of occurrence, which is contrary to previous findings (Omoti and Halim, 2006; Omoti and Imiere, 2006; Nwannadi et al., 2010; Omoti et al., 2012; Kagu et al., 2013; Egesie et al., 2018) that reported varying leukaemia subtypes to be the most frequent haematologic malignancies. Haematologic malignancies have been said to have no particular pattern of occurrence as their occurrence rates can vary with geographical location (Bacher et al., 2005). This could be the reason for the contrary finding. 
Table 3. Relationship between NRAS_G12D and NRAS_G13C mutations, and demographic, clinical features and risk factors of the cancer patients.

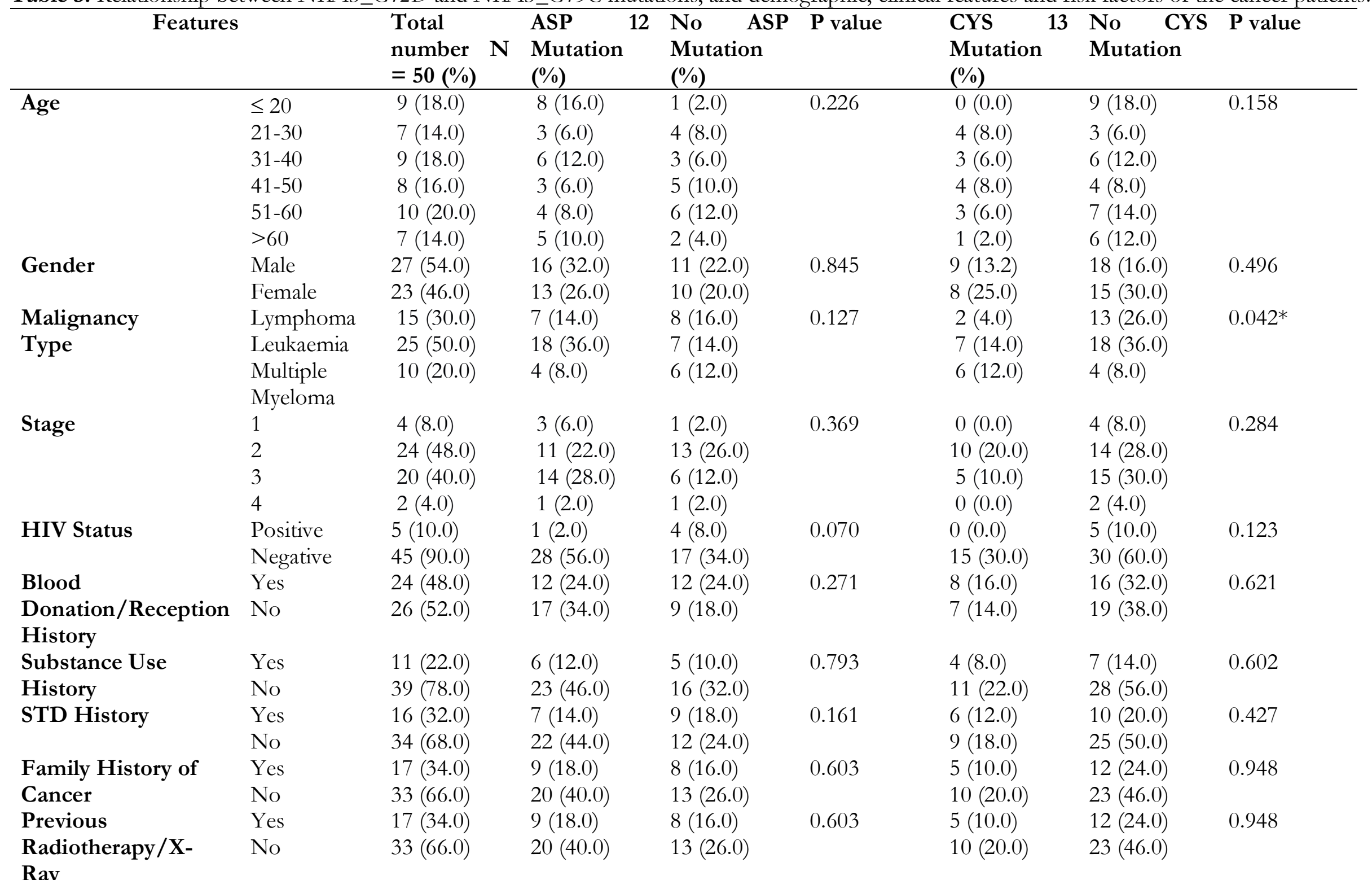

Key: * - Relationship is significant at the 0.05 level (2-sided). 
Table 4. Relationship between NRAS_G12D and NRAS_G13C mutations and demographic and risk factors among blood donors.

\begin{tabular}{|c|c|c|c|c|c|c|c|c|}
\hline \multicolumn{2}{|l|}{ Features } & \multirow{2}{*}{$\begin{array}{l}\text { Total } \\
\text { number } \\
\mathrm{N}=50(\%)\end{array}$} & \multirow{2}{*}{$\begin{array}{ll}\text { ASP } & 12 \\
\text { Mutation } & \\
(\%) & \end{array}$} & \multirow{2}{*}{$\begin{array}{l}\text { No ASP } \\
\text { Mutation }\end{array}$} & \multirow[t]{2}{*}{$\mathbf{P}$ value } & \multirow{2}{*}{$\begin{array}{l}\text { CYS } 13 \\
\text { Mutation } \\
(\%)\end{array}$} & \multirow{2}{*}{$\begin{array}{l}\text { No CYS } \\
\text { Mutation }\end{array}$} & \multirow[t]{2}{*}{$\mathbf{P}$ value } \\
\hline & & & & & & & & \\
\hline \multirow[t]{4}{*}{ Age } & $\leq 20$ & $5(10.0)$ & $1(2.0)$ & $4(8.0)$ & 0.137 & $0(0.0)$ & $5(10.0)$ & 0.332 \\
\hline & $21-30$ & $18(36.0)$ & $1(2.0)$ & $17(34.0)$ & & $1(2.0)$ & $17(34.0)$ & \\
\hline & $31-40$ & $19(38.0)$ & $0(0.0)$ & $19(38.0)$ & & $0(0.0)$ & $19(38.0)$ & \\
\hline & $41-50$ & $8(16.0)$ & $0(0.0)$ & $8(16.0)$ & & $1(2.0)$ & $7(14.0)$ & \\
\hline \multirow[t]{2}{*}{ Gender } & Male & $41(82.0)$ & $1(2.0)$ & $40(80.0)$ & 0.331 & $2(4.0)$ & $39(78.0)$ & 1.000 \\
\hline & Female & $9(18.0)$ & $1(2.0)$ & $8(16.0)$ & & $0(0.0)$ & $9(18.0)$ & \\
\hline Blood & Yes & $30(60.0)$ & $1(2.0)$ & $29(58.0)$ & 1.000 & $1(2.0)$ & $29(58.0)$ & 1.000 \\
\hline $\begin{array}{l}\text { Donation/Reception } \\
\text { History }\end{array}$ & \multicolumn{7}{|c|}{ History } & \\
\hline Substance Use & Yes & $2(4.0)$ & $0(0.0)$ & $2(4.0)$ & 1.000 & $1(2.0)$ & $1(2.0)$ & 0.079 \\
\hline History & No & $48(96.0)$ & $2(4.0)$ & $46(92.0)$ & & $1(2.0)$ & $47(94.0)$ & \\
\hline \multirow[t]{2}{*}{ STD History } & Yes & $2(4.0)$ & $0(0.0)$ & $2(4.0)$ & 1.000 & $1(2.0)$ & $1(2.0)$ & 0.079 \\
\hline & No & $48(96.0)$ & $2(4.0)$ & $46(92.0)$ & & $1(2.0)$ & $47(94.0)$ & \\
\hline Family History of & Yes & $14(28.0)$ & $2(4.0)$ & $12(24.0)$ & 0.074 & $1(2.0)$ & $13(26.0)$ & 1.000 \\
\hline Cancer & No & $36(72.0)$ & $0(0.0)$ & $36(72.0)$ & & $1(2.0)$ & $35(70.0)$ & \\
\hline Previous & Yes & $8(16.0)$ & $1(2.0)$ & $7(14.0)$ & 1.000 & $0(0.0)$ & $8(16.0)$ & 0.297 \\
\hline $\begin{array}{l}\text { Radiotherapy/X- } \\
\text { Ray }\end{array}$ & No & $42(84.0)$ & $1(2.0)$ & $41(82.0)$ & & $2(4.0)$ & $40(80.0)$ & \\
\hline
\end{tabular}

Key: * - Relationship is significant at the 0.05 level (2-sided). 
More males than females had haematopoietic cancers in this study. This corresponds with previous findings (Onwusigwe et al., 2002; Omoti and Halim, 2006; Omoti and Imiere, 2006; Nwannadi et al., 2010; Omoti et al., 2012; Egesie et al., 2018; Anyanwu et al., 2019). The onset of cancer requires both genetic and external (physical, chemical and/or biological) transforming factors, and males tend to be more exposed to the external transforming agents from occupational exposure or their social lifestyle. The genetic composition of the male patients could also play a contributing role, since they have lesser endurance/resistance to unfavourable conditions than the female gender right from the gametic stage. There is therefore a higher chance of having weaker DNA repair machineries than females (Anyanwu et al., 2019). CML and NHL were, however, predominant among the female patients. Even though there was no association between mutation and gender, males than females had NRAS mutations. As previously discussed, the possibility of having weaker DNA repair machineries could be a contributing factor.

Only $4.0 \%$ of the malignant patients from this study had stage 4 cancer. It should be noted that stage 4 is the terminal stage of cancer, as such, fewer patients tend to remain alive with this stage of cancer when related studies are being carried out. Their tendency to die off at the terminal stage may be the reason for the lowest frequency seen in this study. Majority of the cancer patients $(48.0 \%)$ had stage 2 cancer. It would be expected that stage 1 cancer would have a higher frequency than stage 2 cancer. However, conclusive diagnoses are most times not yet achieved at this stage, and sometimes not captured, as clinicians may still be trying out diagnostic protocols on the presumptive patients. Some of these patients may not even report to the hospital until the cancer progresses, which could also be the reason for a lower frequency.

Almost half of the study participants (48.0\%) had NRAS mutations. Majority of these individuals were malignant $(44.0 \%)$, indicating that somatic mutations occur at extremely high rates in cancer patients. Contrary to this finding, previous studies on NRAS mutations in larger cohort of haematologic malignancies like leukaemia showed various frequencies ranging from 6.3 to 12.1\% (Bacher et al., 2005; Liang et al.,
2006; Liang et al., 2018). Only four (4.0\%) of the healthy blood donors had NRAS mutations. Three out of the four blood donors who had the mutated NRAS genes (NRAS_G12D and NRAS_G13C) admitted to having a history of cancer in their family, thus, there might be a likelihood of developing cancer in the future since the oncogenic mutations of the $\mathrm{R} A S$ genes frequently found in human cancers are known to throw the normal outcome of RAS/MAPK signalling pathways off balance, thus leading to tumour development (Fernández-Medarde and Santos, 2011). The fourth donor did not have any history of cancer in his family. However, it was observed from the questionnaire that he admitted to using hard substances such as tobacco, nicotine, heroin and marijuana and medications not prescribed by physicians (such as Tramadol and Codeine). Some of these substances are carcinogenic and could trigger somatic mutations which transform normal cells to cancerous cells. This could explain why he had NRAS_G13C mutation. Illicit drugs like heroin are often mixed with carcinogenic additives called cutting agents. Phenacetin is the most common carcinogenic additive. Benzene, a carcinogen also found in cigarettes, is used in the illicit production of meth. However, somatic mutations have been reported in recent times in normal, non-cancerous cells (Xia et al., 2017; Risques et al., 2018) and associated with old age, although NRAS has not been previously implicated. Then again, the probability of the donor being at risk of some other human ailments should however, not be overruled, as defective $R A S$ signalling has been cited as a contributing factor to many other human illnesses, including diabetes and immunological and inflammatory disorders (Fernández-Medarde and Santos, 2011).

More mutations were observed in G12D $(31.0 \%)$ than G13C $(17.0 \%)$ from this study. The G12D mutation rate obtained was lower than those reported previously (Bacher et al., 2005; Bowen et al., 2005) which found $43.0 \%$ and $43.6 \%$ mutation rates at codon 12 , while G13C mutation rate from this study fell within the range obtained by those studies. The higher mutation rate obtained in G12D is in agreement with previous report that there is generally higher rate of mutation in codon 12 than codon 13 in oncogenic mutation of NRAS genes in human 
haematopoietic tumours (Bacher et al., 2005). The different rates of mutation obtained suggest that different types of NRAS mutations may collaborate better with specific primary mutations in leukemogenesis (Bacher et al., 2005), lymphomagenesis or myelomagenesis. Most reports have linked NRAS mutations to worst prognostic outcome of haematologic malignancies (Paquette et al., 1993; De Melo et al., 1997; Chng et al., 2008; Steinbrunn et al., 2011). G13C mutations have rather been linked to better outcome of leukaemia (Coghlan et al., 1994), while NRAS_G12D has been linked to worse prognostic outcome in haematologic malignancies by initiating uncontrollable activity of ERK, the excessive proliferation of myeloid progenitors, and consequently acute myeloproliferative disease (Wang et al., 2011). The absence of somatic mutation in 6 haematologic cancer individuals in this study is likely because not all blood cancer patients undergo NRAS mutation at G12D and/or G13C. While some undergo mutation at codon 61 (located on exon 3), others do not undergo any mutations. In fact, wild-type NRAS has been confirmed to respond extremely well to monoclonal antibody therapy compared to mutated genes which are known to result in worst prognostic outcome. The patients who do not have NRAS mutant genes may also have mutant RAS genes other than NRAS (e.g KRAS).

The NRAS_G13C mutations were absent among patients below 20 years of age, but were highest among participants aged 21-30 and 41-50 respectively. However, the rate of NRAS_G12D mutations were observed to be highest among participants below the age of 20 . The association between the age of the study participants and the NRAS gene mutation was insignificant $(P>0.05)$. This explains why there was seemingly no pattern established by these mutations in relation to age. While haematologic malignancies may be related to patients' age, oncogenic mutations like NRAS are not age-dependent. The highest rate of mutation in both codons was observed in leukaemia patients. Somatic NRAS mutations have been reported to be commonly observed in aggressive tumours such as leukaemia or multiple myeloma (Le and Shannon, 2002). Findings from this study are in line with this report. The relationship between NRAS_G13C mutation and malignancy type was statistically significant $(\mathrm{p}=$
0.042). Stages 3 and 2 cancer had the highest rates of NRAS_G12D and NRAS_G13C mutations, respectively. The rates of mutation obtained in the two codons as seen in this study can be linked to their prognostic roles. As previously stated, NRAS_G12D is associated with worst outcome, hence its increased rate in the more advanced stage of cancer, while NRAS_G13C is associated with better outcome, hence a higher rate is seen in less advanced stage. As previously discussed also, lower mutation rates in the terminal and initial stages of cancer could be due to lower number of captured cases. No relationship was observed between both NRAS mutations found in the cancer patients and the related risk factors $(P>$ 0.05). This is not surprising as majority of the patients were not exposed to the analysed risk factors at the time of the study.

Few blood donors had NRAS_G12D and NRAS_G13C mutations, and there was no relationship between the age and gender of the blood donors and the occurrence of these mutations $(P>0.05)$. There was also no association between NRAS mutations and the donors' history of blood donation/transfusion and radiotherapy/X-ray, family history of cancer, previous history of substance abuse and STD. Nonetheless, since increased and frequently occurring NRAS mutation in healthy cells may lead to their oncogenic transformation, NRAS mutation in these donors is a probable indicator of possible malignancy in the future.

Sequencing analysis revealed NRAS G12D (c.35 GGT >GAT) mutation among study participants but NRAS G13C mutation was not observed. It has been said that mutations which are detected by AS-PCR may not always be observed via sequencing because sequencing is not exquisitely sensitive (Vasioukhin et al., 1996; Ellison et al., 2011). This could be the reason for the absence of NRAS G13C mutation via sequencing from this study. A mutation must be present in approximately $20 \%$ of the sample to be readily detected ( $\mathrm{Li}$ et al., 2008). Other cancer studies have found the percentage mutation in a tumour sample to be as low as $6 \%$, significantly lower than sequencing is able to detect (Ellison et al., 2011; Fox et al., 1998). As such, given the heterogeneity of tumours (Taniguchi et al., 2008), the percentage could possibly be even lower in 
some tumour blood product samples like sera or plasma.

Unlike sequencing, multiplex PCR techniques such as AS-PCR and ARMS assays only detect the mutations they were designed to interrogate. However, this could be considered an advantage in a clinical setting so that decisions on treatment or patient-outcome results are based only on known, clinically validated mutations (Ellison et al., 2011).

Deregulation of $R A S$-dependent signalling is the essential requirement for tumorigeneses (Anyanwu et al., 2019). Overall, studies on NRAS mutation would pave way for better understanding of patients' cases and prognostic outcome, subsequently providing more leverage for development of suitable monoclonal antibody therapy for cancer treatment.

\section{CONCLUSION}

Our study found a higher rate of haematologic malignancies in male than females. The highest rate of cancer was seen among male patients with ALL and MM and female patients with CML and NHL (10\% respectively). Stage 2 cancer had the highest frequency of $24.0 \%$. Mutations observed in this study were higher in codon $12(31.0 \%)$ than codon $13(17.0 \%)$. The highest rate of mutation was observed in leukaemia patients, having a significant association with codon 13. Stages 3 and 2 had the highest rate of mutation for NRAS_G12D and NRAS_G13C each. In view of these findings, it was established that NRAS mutations increase the susceptibility of the enrolled individuals to haematologic malignancies, and also progression of the malignancies. Hence, these mutations play potentially predictive role in the prognosis of haematologic malignancies in Nigerian setting, as is the case worldwide.

\section{ETHICAL APPROVAL}

Informed consent was obtained from the cohort, and the participants were enrolled consecutively. Ethical consent for this study was sought from the
Research Ethics Committees in charge of the selected hospitals. Reference numbers of approval are: NHA/ADMIN/236/V.VII (National Hospital, Abuja), FCT/UATH/HREC/PR/538 (University of Abuja Teaching Hospital), FHREC/2016/01/52/20-07-16 (Garki Hospital Abuja).

\section{REFERENCES}

Anyanwu, N. C. J, Ella, E. E., Aminu, M., \& Kazeem, H. M. 2019. Detection of NRAS_G12D and NRAS_G13C mutant genes among apparently healthy and haematologic malignant individuals in Federal Capital Territory, Nigeria, Journal of Immunoassay and Immunochemistry 40(6): 605-616.

Bacher, U., Haferlach, T., Schoch, C., Kern, W., \& Schnittger S. 2005. Implications of NRAS mutations in AML: a study of 2502 patients. Blood 107(10): 3847-3853.

Bowen, D. T., Frew, M. E., Hills, R., Gale, R. E., \& Wheatley K. 2005. RAS mutation in acute myeloid leukemia is associated with distinct cytogenetic subgroups but does not influence outcome in patients younger than 60 years. Blood 106: 2113 2119.

Coghlan, D. W., Morley, A. A., Matthews, J. P., \& Bishop, J. F. 1994. The incidence and prognostic significance of mutations in codon 13 of the NRAS gene in acute myeloid leukemia. Leukemia 8(10): 1682-7.

Chng, W. J., Gonzalez-Paz, N., Price-Troska, T., Jacobus, S., Rajkumar, S. V., Oken, M. M., Kyle, R. A., Henderson, K. J., Van Wier, S., Greipp, P., Van Ness, B., \& Fonseca, R. 2008. Clinical and biological significance of RAS mutations in multiple myeloma. Lenkemia 22(12): 2280-2284

De Melo, M. B., Lorand-Metze, I., Lima, C. S., Saad, S. T., \& Costa, F. F. 1997. NRAS gene point mutations in Brazilian acute myelogenous leukemia patients correlate with a poor prognosis. Leuk Lymphoma 24(3-4): 309-17.

Egesie, O. J., Jatau, E. D., Damulak, O. D., Zakari, A., Jasini, J., Akinola, O. I., Adeyemi, O., Akor, E., \& Egesie, U. G. 2017. Prevalence and type of haematological malignancies among adults in a tertiary hospital in Jos-Nigeria: a sixteen-year retrospective analysis. Highland Medical Research Journal 17(2).

Egesie, O. J., Agaba, P. A., Silas, O. A., Achenbach, C., Zoakah, A., Agbaji, O., Madaki, J. A., Okeke, E. N., Hou, L., Sagay, A. A., \& Murphy, R. 2018. Presentation and survival in patients with hematologic malignancies in Jos, Nigeria: A retrospective cohort analysis. Journal of Tropical Medicine 20: 49-56.

Ellison, G., Donald, E., McWalter, G., Knight, L., Fletcher, L., Sherwood, J., Cantarini, M., Orr, M., \& Speake, G. 2011. A comparison of ARMS and DNA sequencing for mutation analysis in clinical biopsy samples. Journal of Experimental Clinical Cancer Research 29(1): 132.

Fernández-Medarde, A. \& Santos, E. 2011. Ras in Cancer and Developmental Diseases. Genes and Cancer 2(3): 344-358.

Fox, J. C., England, J., White, P., Ellison, G., Callaghan, K., Charlesworth, N. R., Hehir, J., McCarthy, T. L., SmithRavin, J., Talbot, I. C., Snary, D., Northover, J. M., Newton, C. R., \& Little, S. 1998. The detection of K-ras mutations in colorectal cancer using the amplification-refractory mutation system. British Journal of Cancer 77(8): 1267-74.

Global Burden of Disease Cancer Collaboration. 2017. Global, regional, and national cancer incidence, mortality, years of 
life lost, years lived with disability, and disability-adjusted life-years for 32 cancer groups, 1990 to 2015: A systematic analysis for the global burden of disease study. JAMA Oncology 3: 524-48.

Hamid, G. A. 2012. The pattern of haematological malignancies at the AlGamhouria Teaching Hospital, Aden, Yemen, from 2008 to 2010. Turkish Journal of Hematology 29: 342-7.

Janssen, J. W., Steenvoorden, A. C., Lyons, J., Anger, B., Böhlke, J. U., Bos, J. L., Seliger, H., \& Bartram, C. R. 1987. RAS gene mutations in acute and chronic myelocytic leukemias, chronic myeloproliferative disorders, and myelodysplastic syndromes. Proceedings of the National Academy of Sciences US A 84(24): 9228-9232.

Kagu, M. B., Ahmed, S. G., Bukar, A. A., Mohammed, A. A., Mayun, A. A., \& Musa, A. B. 2013. Spectrum of haematologic malignancies and survival outcomes of adult lymphomas in Maiduguri, northeastern Nigeria - A fourteen-year review. African Journal of Medicine and Medical Sciences 42: 5-14.

Le, D. T. \& Shannon, K. M. 2002. Ras processing as a therapeutic target in hematologic malignancies. Current Opinions in Hematology 9(4): 308-315.

Li, J., Wang, L., Mamon, H., Kulke, M. H., Berbeco, R., \& Makrigiorgos, G. M. 2008. Replacing PCR with COLDPCR enriches variant DNA sequences and redefines the sensitivity of genetic testing. Nature Medicine 14(5): 579-84.

Liang, D. C., Shih, L. Y., Fu, J. F., Li, H. Y., Wang, H. I., Hung, I. J., Yang, C. P., Jaing, T. H., Chen, S. H., \& Liu, H. C. 2006. $\mathrm{K}$-Ras mutations and NRAS mutations in childhood acute leukemias with or without mixed-lineage leukemia gene rearrangements. Cancer 106: 950-956.

Liang, D. C., Chen, S. H., Liu, H. C., Yang, C. P., Yeh, T. C., Jaing, T. H., Hung, I. J., Hou, J. Y., Lin, T. H., Lin, C. H., \& Shih, L. Y. 2018. Mutational status of NRAS, KRAS, and PTPN11 genes is associated with genetic/cytogenetic features in children with B-precursor acute lymphoblastic leukemia. Pediatric Blood \& Cancer 65: e26786.

McCubrey, J. A., Steelman, L. S., Abrams, S. L., Lee, J. T., Chang, F., Bertrand, F. E., Navolanic, P. M., Terrian, D. M., Franklin, R. A., D’Assoro, A. B., Salisbury, J. L., Mazzarino, M. C., Stivala, F., \& Libra, M. 2006. Roles of the $\mathrm{RAF} / \mathrm{MEK} / \mathrm{ERK}$ and PI3K/PTEN/AKT pathways in malignant transformation and drug resistance. Advances in Ensyme Regulation 46: 249-279.

Nwannadi, I. A., Alao, O. O., Bazuaye, G. N., \& Halim, N. K. 2010. The epidemiology of haematological malignancies at the University of Benin Teaching Hospital: A ten-year retrospective study. Internet Journal f Epidemiology 9.

Omoti, C. \& Halim, N. K. 2006. Plasma cell myeloma in a tertiary centre in the niger delta region of nigeria: clinicoimmunologic analysis. Pakistan Journal of Medical Sciences 23(1): 27-32.

Omoti, C. E. \& Imiere, E. O. 2006. Trends in the pattern of leukaemia incidence in a tertiary health care centre in Nigeria: 1990-2004. Journal of Medicine and Biomedical Research 5(2): 44-49.

Omoti, C. E., Nwannadi, I. A., Obieche, J. C., \& Olu-Eddo, A. N. 2012. The epidemiological features of lymphoid malignancies in Benin City, Nigeria: A15 years study. The Pan African Medical Journal 11: 10.

Onwuasigwe, C. N., Aniebue, P. N., \& Ndu, A. C. 2002. Spectrum of Paediatric Malignancies in Eastern Nigeria (1989-1998). West African Journal of Medicine 21(1): 31-33.

Paquette, R. L., Landaw, E. M., Pierre, R. V., Kahan, J., Lubbert, M., Lazcano, O., Isaac, G., McCormick, F., \& Koeffler, H. P. 1993. NRAS mutations are associated with poor prognosis and increased risk of leukemia in myelodysplastic syndrome. Blood 82(2): 590-9.
Risques, R. A., \& Kennedy, S. R. 2018. Aging and the rise of somatic cancer-associated mutations in normal tissues. PLoS Genetics 14(1): e1007108.

Steinbrunn, T., Stuhmer, T., Gattenlohner, S., Rosenwald, A., Mottok, A., Unzicker, C., Einsele, H., Chatterjee, M., \& Bargou, R. C. 2011. Mutated RAS and constitutively activated Akt delineate distinct oncogenic pathways, which independently contribute to multiple myeloma cell survival. Blood 117(6): 1998-2004.

Taniguchi, K., Okami, J., Kodama, K., Higashiyama, M., \& Kato, K. 2008. Intratumour heterogeneity of epidermal growth factor receptor mutations in lung cancer and its correlation to the response to gefitinib. Cancer Science 99(5): 929-35.

van 't Veer L. J., Burgering, B. M., Versteeg, R., Boot, A. J., Ruiter, D. J., Osanto, S., Schrier, P. I., \& Bos, J. L. 1989. N-ras mutations in human cutaneous melanoma from sunexposed body sites. Molecular and Cellular Biology 9(7): 31143116.

Vasioukhin, V., Anker, P., Maurice, P., Lyautey, J., Lederrey, C., \& Stroun, M. 1996. Point mutations of the N-ras gene in the blood plasma DNA of patients with myelodysplastic syndrome or acute myelogenous leukaemia. British Journal of Haematology 86: 774-9.

Wang, J., Liu, Y., Li, Z., Wang, Z., Tan, L.X., Ryu, M-J., Meline, B., Du, J., Young, K.H., Ranheim, E., Chang, Q., \& Zhang J. 2011. Endogenous oncogenic Nras mutation initiates hematopietic malignancies in a dose- and cell typedependent manner. Blood 118(2): 368-379.

World Health Organisation. 2019. Cancer: Key Statistics. Retrieved from https://www.who.int/cancer/resources/ keyfacts/en/

Xia, L., Li, Z., Zhou, B., Tian, G., Zeng, L., Dai, H., Li, X., Liu, C., Liu, C., Lu, S., Xu, F., Tu, X., Deng, F., Xie, Y., Huang, W., \& He, J. 2017. Statistical analysis of mutant allele frequency level of circulating cell-free DNA and blood cells in healthy individuals. Scientific Reports 7: 7526. 